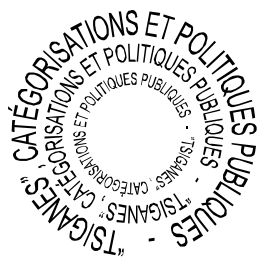

\title{
MIGRANTS ROMS DANS L'ESPACE PUBLIC : (IN)VISIBILITÉS CONTRAINTES
}

\author{
Marion DALIBERT * \\ Milena DOYTCHEVA **
}

Depuis le début des années 2000 et plus particulièrement depuis 2002, périodes de libéralisation des régimes de circulation pour les ressortissants roumains et bulgares, et 2007, année de l'adhésion de la Roumanie et de la Bulgarie à l'Union européenne, les migrations dites "roms" qui s'intensifient vers la France et vers d'autres pays européens occupent une place importante dans le débat public et à l'intérieur de l'espace politique. Identifiées comme Roms, Tsiganes (mais aussi Gitans, Sinti, Manouches...), ces populations romani sont l'objet de polémiques, de vives tensions politiques ainsi que de mobilisations importantes sur la scène locale, dans les champs associatif ou militant.

Alors qu'ils sont peu nombreux - leur nombre est estimé entre 15000 et $20000^{1}$ - leur présence sur le territoire français fait l'objet d'un important traitement médiatique. C'est ce traitement médiatique que notre contribution se propose d'explorer, en attachant une attention particulière à la manière dont il participe de la production et de la mise en circulation de discours définitoires et de représentations. En interrogeant plus particulièrement les modalités selon lesquelles se construisent dans la sphère publique - matérialisée ici par les grands médias - tes figures collectives des Roms, nous ferons l'hypothèse que ces populations immigrées se voient simultanément représentées en tant que groupe et collectivité, processus dans lequel la dimension ethno-culturelle, mais aussi ethno-raciale occupent une place importante.

* Docteure en sciences de l'information et de la communication, enseignante, Groupe d'études et de recherche interdisciplinaire en information communication (GERIICO), Université de Lille 3.

** Sociologue, maître de conférences, Délégation CNRS, Centre d'analyse et d'intervention sociologiques (CADIS) - École des hautes études en sciences sociales (EHESS), Université de Lille 3.

1. Dont 750 personnes environ bénéficiant de dispositifs spécifiques d'hébergement et d'“insertion". Voir COLLECTIF NATIONAL DROITS DE L'HOMME ROMEUROPE, Rapport sur la situation des Roms migrants en France, 2009-2010, Paris : Collectif national Droits de l'homme Romeurope, septembre 2010, 153 p. (voir pp. 82 et ss.), http://www.romeurope.org/IMG/ Rapport\%20Romeurope\%202009-2010.pdf 
Alors même qu'en France la catégorie "gens du voyage", dont ils sont à tort rapprochés, est soumise depuis les années 1960 à un traitement bureaucratique et administratif visant entre autres à sa " désethnicisation ", ces migrants venus de l'Est sont l'objet d'un processus de catégorisation dans la sphère publique qui les ethnicise et altérise à la fois.

Selon quelles logiques la construction de ces figures collectives des Roms - mais aussi de "la question rom", du "problème rom" opère-t-elle dans les représentations médiatiques? Comment, plus particulièrement, la saillance d'une catégorisation à dimension ethnique et raciale se combine-t-elle au credo républicain de l'indivisibilité de la nation et de la cécité quant aux origines? Telles sont certaines des questions que nous proposons d'aborder à partir d'une étude de la représentation médiatique au sein d'un corpus d'articles issu de la presse quotidienne nationale dite "de référence" (Le Figaro, Le Monde, Libération) $)^{2}$. Dans une perspective constructiviste d'analyse de discours, nous nous attachons à rendre compte de la manière dont se construit l'identité socio-discursive de ces groupes et individus, la notion d'identité socio-discursive étant entendue comme le produit d'actes d'identification et de catégorisation portés sur un groupe social ou des individus, et qui sont mis en scène dans le discours médiatique, opérés par les journalistes ou par des personnes dont la parole est rapportée dans les médias ${ }^{3}$.

\section{Migrants roms dans l'espace public: du politique au médiatique}

Comme le titre la revue Lignes qui, en 2011 , consacre deux numéros à la question, "L'exemple des Roms. Les Roms, pour l'exemple", , ces migrations se trouvent prises aujourd'hui dans des processus complexes

2. Nous avons collecté les articles du corpus de 2008 à 2012 en utilisant le mot-clé "roms", ce qui fait en totalité 650 articles. Ces populations occupent régulièrement l'espace médiatique lors des périodes estivales : elles font l'objet, respectivement, de 34, 379 et 149 articles au cours des mois de juillet, août et septembre 2008, 2010 et 2012, périodes de référence que nous avons sélectionnées en priorité pour l'analyse.

3. Cf. DALIBERT, Marion, Accès à l'espace public des minorités ethnoraciales et "blanchité". La construction du Sujet de la Nation française dans la médiatisation de Ni putes ni soumises et du Mouvement des Indigènes de la République dans la presse quotidienne nationale dite "de référence" (Le Figaro, Le Monde, Libération) et dans les journaux télévisés de TF1, France 2 et France 3, thèse de doctorat en sciences de l'information et de la communication, Université de Lille 3, $615 \mathrm{p}$.

4. CANUT, Cécile (dossier coordonné par), "L'exemple des Roms. Les Roms, pour l'exemple", Lignes, $\mathrm{n}^{\circ} 34$, février 2011, 192 p. ; CANUT, Cécile (dossier coordonné par), "L'exemple des Roms. Les Roms, pour l'exemple", Lignes, ${ }^{\circ} 35$, juin 2011, où est publié la suite du dossier. 
de représentations, d'imbrication d'idéologies et de projections qui mettent en scène des enjeux emblématiques pour les démocraties contemporaines ayant trait aux questions de citoyenneté, de solidarité, d'enracinement et de mobilité dans une Europe qui se voudrait sans frontières.

C'est à l'été 2010, dans un contexte particulièrement répressif en matière d'immigration, que se développe en France une première polémique qui vise les Roms et qui résonne sur le plan politique tant national qu'européen ${ }^{5}$. Alors qu'ils sont citoyens de l'Union européenne, les Roms sont depuis plusieurs années comptabilisés dans les chiffres officiels des expulsions du territoire français dont ils représentent environ le tiers $^{6}$. Si la directive 2004/38/CE relative au droit des citoyens de circuler et de séjourner librement sur le territoire des États membres encadre dans des limites très strictes la possibilité d'éloignement, à partir de l'année 2006 plusieurs mesures et dispositifs ad hoc sont introduits en droit français visant à "illégaliser" ces migrants (souvent à les "criminaliser") et à les rendre plus facilement "expulsables"7.

À partir de l'année 2010, un pas supplémentaire est franchi dans cette logique avec la définition explicite des populations incriminées et, en l'occurrence, la mention expresse des " Roms ॥. Des objectifs chiffrés en matière d'évacuation de « campements illicites [...], en priorité ceux des Roms $1{ }^{8}$, sont donnés, alors que les charters de l'été 2010 marquent l'opinion française ainsi que l'image de la France à l'étranger. Dans un contexte politique renouvelé par l'alternance présidentielle et législative

5. Voir notamment la réunion interministérielle du 28 juillet 2010 à l'Élysée, lorsque le gouvernement déclare vouloir s'attaquer aux « problèmes que pose le comportement de certains des gens du voyage et des Roms", suivie du discours dit de Grenoble du président de la République Nicolas Sarkozy prononcé le 30 juillet 2010 dans cette ville à l'occasion de la prise de fonction du nouveau préfet de l'Isère. Dans la foulée, une circulaire du ministère de l'Intérieur du 5 août 2010 invite les préfets à engager le démantèlement "systématique des camps illicites, en priorité ceux des Roms", et fixe des objectifs chiffrés d'évacuation et d'expulsion : "Les préfets [...] s'assureront [...] de la réalisation minimale d'une opération importante par semaine (évacuation/démantèlement/reconduite) concernant prioritairement les Roms». Qualifiant l'attitude de la France de "honteuse », la Commission européenne, par la voix de Viviane Reding, en charge de la Justice, des Droits fondamentaux et de la Citoyenneté (discours du 14 septembre 2010), entend ouvrir une procédure d'infraction à la législation européenne.

6. COMMISSION NATIONALE CONSULTATIVE DES DROITS DE L'HOMME, Étude et propositions sur la situation des Roms et des gens du voyage en France, Paris : $\mathrm{CNCDH}$, février 2008, 66 p., http://www.cncdh.fr/sites/default/files/08.02.07_etude_sur_la_situation_des_roms_ et_des_gens_du_voyage_en_france.pdf

7. GROUPE D'INFORMATION ET DE SOUTIEN DES IMMIGRÉS, Plainte contre la France pour violation du droit communautaire en matière de libre circulation des personnes, Paris : GISTI, juillet 2008, 43 p., http://www.gisti.org/IMG/pdf/saisine_com-eur_2008-07-31_lib-circ.pdf

8. MINISTÈRE DE L'INTÉRIEUR, Circulaire du 5 août 2010 concernant l'évacuation des campements illicites. 
intervenue en 2012, les objectifs sécuritaires et répressifs en matière de politique publique persistent. Après une parenthèse ${ }^{9}$, la gauche prolonge sans réserve les politiques d'expulsion entamées par ses prédécesseurs depuis 2002 : en témoignent l'“affaire Leonarda", largement médiatisée, tout comme le "ils ne souhaitent pas s'intégrer " de Manuel Valls, à la rentrée politique de septembre 2013, faisant le constat d'un " mode de vie " qui serait spécifique, en " confrontation " avec la société française ${ }^{10}$.

Les logiques d'altérisation et de marquage ethno-racial trouvent une illustration éloquente dans le corpus de données constitué à partir de l'étude de la presse, notamment parce que les médias font office de relais entre la sphère politique et l'opinion publique. Le concept de marquage social est ici emprunté à Wayne Brekhus pour désigner "I les manières dont les acteurs sociaux perçoivent activement une des faces d'un contraste tout en ignorant l'autre face, conçue comme épistémologiquement non problématique $1{ }^{11}$. Les individus marqués socialement sont généralement catégorisés par des attributs identitaires "problématiques": les femmes marquées par la race ne sont pas désignées uniquement par le terme "femmes", mais par des syntagmes signifiant une origine spécifique : "femmes issues de l'immigration", "femmes d'origine sénégalaise ", etc.

Par l'usage de syntagmes spécifiques, les producteurs de discours différencient les populations ciblées d'une majorité considérée comme neutre et générique. Comme le propose Colette Guillaumin, l'll existence [des groupes majoritaires] ne se saisit que par l'absence de limitation en face des groupes catégorisés qui sont étroitement déterminés. Alors que la situation majoritaire se décline comme une forme de liberté dans la définition de soi, celle-ci n'est jamais reconnue au minoritaire dont l'appartenance se définirait d'abord comme ce que ce dernier ne peut se donner à soi-même $11^{12}$. Ce processus de marquage social et de catégorisation prend ici la forme, comme nous le verrons, d'une altérisation, attendu qu'il produit une distinction entre un "nous" et un "Autre" qui en est exclu.

9. Marquée par exemple par la circulaire du 26 août 2012 qui prévoit des mesures d'accompagnement dans l'évacuation des campements "illicites". La circulaire fut toutefois peu appliquée.

10. Interview donnée à France Inter le 24 septembre 2013.

11. BREKHUS, Wayne, "Une sociologie de l'invisibilité' : réorienter notre regard", Réseaux, $\mathrm{n}^{\circ}$ 129130, 2005, pp. 243-272 (voir p. 246).

12. GUILLAUMIN, Colette, L'idéologie raciste : genèse et langage actuel, Paris : Éd. Mouton, 1972, 248 p. (voir p. 196). 


\section{- Un groupe hyper-(in)visible}

La construction de ces frontières symboliques, sociales et politiques entre un "Autre" - les populations "roms" - et le "nous" national, portée par les politiques, fait écho aux représentations médiatiques de ces populations. La quasi-totalité des articles analysés du corpus rend compte d'interrogations concernant leur présence en France et plus généralement en Europe occidentale. Les sujets polémiques et de débat prédominent, avec une focale importante placée sur des actions et des mesures répressives qui ne manquent pas d'offrir l'occasion d'évoquer les spectres de la menace, du problème ou de l'insécurité.

L'étude de la presse a également montré que ces questions occupent l'espace médiatique de manière privilégiée lors des périodes estivales. Ainsi, les mois de juillet, d'août et de septembre constituent des pics dans leur événementialisation : sur les 650 articles du corpus, 562 ont été publiés les étés 2008,2010 et 2012 . De juillet à septembre 2008 , le cadrage médiatique du "problème rom" fut territorialisé majoritairement en Italie, le gouvernement italien ayant décidé de ficher les habitants des camps; à l'été 2010 , période du corpus qui compte le plus d'articles (379 sur 650), la controverse se déplace vers la France avec les actions et les interventions du président Nicolas Sarkozy et de son gouvernement qui annoncent vouloir s'attaquer aux " problèmes posés " par " les comportements de certains parmi les gens du voyage et les Roms $1{ }^{13}$. Enfin, à l'été 2012 , ce sont les expulsions demandées par le ministre de l'Intérieur, Manuel Valls, qui focalisent les débats.

Cette hypervisibilité, notamment lors des périodes estivales, est significative, paradoxalement, d'une invisibilité sociale pour ces migrants: étant très peu décrits ou interrogés, n'ayant pas de visage, ils n'existent quasiment que par la désignation. En effet, à l'extérieur du débat politique, les Roms apparaissent peu dans les médias. Les journalistes se sont rarement déplacés pour rendre compte, au sein de genres journalistiques "authentifiants" ", des problématiques quotidiennes auxquelles ils doivent faire face.

13. Réunion interministérielle du 28 juillet 2010. Voir note 5.

14. La collecte de témoignages que l'on peut rapprocher de ce que Michel Foucault appelle « la procédure de l'aveu" constitue en effet un mode important de "production de vérité" ou "d'authentification» des phénomènes sociaux. Voir FOUCAULT, Michel, Histoire de la sexualité, 1 : La volonté de savoir, Paris : Éd. Gallimard, 1991, 211 p. ; DALIBERT, Marion, Accès à l'espace public des minorités ethnoraciales et "blanchité", op. cit. 
À l'exception de 15 reportages parus dans Libération et Le Monde - ce qui représente environ $3 \%$ des articles du corpus - les Roms ont été peu décrits et présentés. Surtout, ils n'ont guère eu accès à la parole publique, y compris dans ces 15 reportages, sauf lorsque les articles rendent compte de leur situation dans un pays étranger. Les seuls acteurs sociaux qui ont pu porter un discours en leur nom furent des associations d'aide ou des représentants de l'Église. En revanche, les principaux intéressés y ont eu un accès limité. Leurs revendications ou points de vue n'ont pas été reconnus comme dignes de valeur sociale ou considérés comme légitimes dans les médias, ce qui est significatif de la position subalterne qui leur est assignée $e^{15}$.

\section{Une minorité altérisée}

$\mathrm{Ce}$ "groupe sans visage", construit dans et par le regard d'autres acteurs sociaux, a néanmoins été culturellement et racialement défini et identifié dans la presse en écho aux discours tenus par les politiques. Nous pouvons distinguer plus particulièrement deux opérateurs matérialisant la représentation médiatique d'une altérité essentialisée et racisée $^{16}$ : l'affiliation constante à un territoire et le marquage ethnoracial par le genre.

\section{- L'assignation par le territoire}

Dans le discours médiatique, les populations désignées par le terme "Roms", et plus rarement "Tsiganes", sont souvent rapprochées d'une territorialité spécifique, généralement un camp, une "aire d'accueil" ou un "village d'insertion". Les Roms ne sont mis en scène qu'à l'intérieur de zones dans lesquelles ils se trouvent confinés décrites comme étant différentes du reste du territoire national : leur lieu d'habitation est extérieur à l'espace urbain, situé le plus souvent en banlieue, entre une voie ferrée et une sortie d'autoroute. Par exemple, une journaliste du Monde en déplacement dans un camp à Choisy-le-Roi (Val-de-

15. Cf. SPIVAK, Gayatri Chakravorty, Les subalternes peuvent-elles parler ? Paris : Éd. Amsterdam, 2009, 109 p.

16. Nous entendons ici la notion de "racialisation" ou de "racisation" comme le processus d'imputation raciale qui consiste à absolutiser la différence culturelle et "d'origine" en l'instituant comme "race", c'est-à-dire en l'inscrivant dans la nature. C'est un rapport social qui lie racisant et racisé dans des rapports de pouvoir où le second est placé en position subordonnée par rapport au premier. Voir DE RUDDER, Véronique ; POIRET, Christian ; VOURC'H, François, L'inégalité raciste : l'universalité républicaine à l'épreuve, Paris : Presses universitaires de France, 2000, 213 p. (voir p. 32). 
Marne) a dépeint II une vingtaine de familles roms [qui] vivotent sur un ancien parking situé au ras des voies ferrées. Les caravanes fatiguées tremblent toutes les trois minutes au souffle du RER $1{ }^{17}$.

Nous pouvons rapprocher ce phénomène de "marquage spatial" - ou de marquage par l'espace - de la représentation médiatique de la "banlieue" analysée dès les années 1980 comme un espace racialisé $^{18}$. En effet, comme le met en avant Édouard Mills-Affif, les zones géographiques reliées aux minorités dans les médias rendent toujours compte d'un fossé entre les personnes qui y vivent et les membres (types) de la communauté nationale : "I Du bidonville au HLM, le décor n'est plus le même mais les représentations restent identiques. II s'agit toujours d'un espace confiné, présenté comme une zone de non-droit, d'une enclave à l'écart de la civilisation $1{ }^{19}$. À l'image des habitants de ces quartiers populaires, les Roms sont toujours représentés en rapport à un espace stigmatisé, appartenant à la marge de la ville et de la société. Cependant, dans le cas de ces migrants, leur lieu d'habitation est dépeint également par les journalistes comme une II zone insalubre 11 , misérable et surpeuplée.

À la représentation médiatique de l'excentrement s'ajoute ainsi celle d'une certaine II souillure "I au sens de Mary Douglas, où les métaphores de la pollution, les rappels de la saleté et du désordre signalent l'impureté et "la marge $\|^{20}$. La presse quotidienne a beaucoup décrit l'insalubrité des zones d'habitation, à l'image de cet extrait d'un article publié dans Libération : "I À l'intérieur d'une ancienne imprimerie s'entassaient 106 Roms sur les $300 \mathrm{~m}^{2}$ que compte la bâtisse. Ils y vivaient depuis la fin de l'année 2007, sans eau courante "pendant six mois", rapporte une voisine. Un endroit insalubre, dépourvu de toilettes. Des mètres cubes de détritus au milieu de la cour permettaient de se cacher pour faire ses besoins $1{ }^{21}$. Les conditions de logement extrêmement précaires, avec

17. VINCENT, Élise, "À Choisy, le lent et délicat pari de l'intégration des Roms", Le Monde du 2-92012, http://www.lemonde.fr/societe/article/2012/09/02/a-choisy-le-lent-et-delicat-pari-de-Iintegration-des-roms_1754419_3224.html

18. Cf. BOYER, Henri ; LOCHARD, Guy, Scènes de télévision en banlieues, 1950-1994, Paris : Éd. L'Harmattan - INA, 1998, 201 p. ; HANCOCK, Claire, "Décoloniser les représentations : esquisse d'une géographie culturelle de nos 'Autres'", Annales de géographie, $\mathrm{n}^{\circ}$ 660-661, 2008, pp. 116-128.

19. MILLS-AFFIF, Édouard, Filmer les immigrés : les représentations audiovisuelles de l'immigration à la télévision française. 1960-1986, Bruxelles : Éd. De Boeck - INA, 2004, 299 p. (voir p. 32).

20. DOUGLAS, Mary, Purity and danger : an analysis of the concepts of pollution and taboo, New York : Routledge Publishers, 1992, 188 p. Voir aussi PICKER, Giovanni, "Gypsies in Pescara : blaming and the politics of silence", Tours, 24-25 mars 2011.

21. COGNÉ, Gaël, “L'errance des Roms de squat en squat”, Libération du 1-8-2008, http://www.liberation.fr/france/2008/08/01/l-errance-des-roms-de-squat-en-squat_77324 
une référence récurrente à un univers de "détritus " et de menaces "d'infestation " ont été également mises en avant dans Le Monde : "I Sunita [enfant rom] vit dans un squat de Vitry-sur-Seine (Val-de-Marne) surpeuplé, infesté de rats, que les pouvoirs publics ont décidé de rayer de la carte 1$)^{22}$.

On retrouve ces représentations de la "mise à l'écart" avec les politiques locales d'"insertion". Entreprises par plusieurs municipalités depuis l'adhésion de la Roumanie et de la Bulgarie à l'Union européenne en 2007, ces politiques visent à offrir aux résidents des bidonvilles une alternative à l'évacuation pure et simple, au moins en termes d'hébergement et de logement. Ces "villages d'insertion" sont par ailleurs mis en scène dans la presse dans leur situation d'excentrement et de ségrégation urbaine. Libération, qui a consacré plusieurs articles à ce sujet, décrit le village d'Aubervilliers comme II voisin des abattoirs de la ville, situé sous l'A86 derrière la cité des 4000 " $^{23}$, comptant II une vingtaine de logements identiques sur 3500 mètres carrés, au bord de l'autoroute et de la voie ferrée, depuis sa création en $200711^{24}$. Ces lieux d'habitation — régulés par les pouvoirs publics sont néanmoins décrits par les journalistes comme étant plus organisés, plus " propres ").

Parallèlement à cette affiliation ou assignation territoriale, les femmes sont présentées dans la presse comme contraintes de mendier, tandis que les hommes sont mis en scène dans des travaux temporaires, non déclarés, collectant des métaux pour les revendre. Libération a par exemple mis en avant que "I la plupart des femmes passent quelques heures à mendier " tandis que "les hommes se partagent des plans boulots $\|^{25}$ et a dépeint le quotidien de "Léonard [qui], à l'instar de la plupart des Roms [...], est ferrailleur ${ }^{26}$. Les seules personnes décrites comme travaillant légalement sont les résidents des "villages d'insertion": " Dans son canapé de velours vert, Argentina, la quarantaine, s'accorde un moment de répit après sa journée de travail. Comme douze

22. SIMON, Catherine, "Roms : la vie devant soi", Le Monde du 13-7-2010, http://www.lemonde.fr/ societe/article/ 2010/07/12/roms-la-vie-devant-soi_1386876_3224.html

23. ANDRÉ, Pauline, "À Aubervilliers, la caravane trépasse", Libération du 29-7-2010, http://www.liberation.fr/societe/2010/07/29/a-aubervilliers-la-caravane-trepasse_669130

24. SOULÉ, Véronique, "Aubervilliers : des Roms à l'école de l'insertion", Libération du 9-9-2010, http://www.liberation.fr/societe/2010/09/09/aubervilliers-des-roms-a-l-ecole-de-l-insertion_677600

25. AUFFRAY, Élodie, "Un dimanche avec des familles en transit dans un bois du Val-de-Marne", Libération du 14-9-2010, http://www.liberation.fr/societe/2010/09/14/roms-vies-ouvertes_678656

26. CHAHINE, Marwan, "À Saint-Denis, les Roms expulsés se réinstallent", Libération du 11-8-2010, http://www.liberation.fr/societe/2010/08/11/a-saint-denis-les-roms-expulses-se-reinstallent 671386 
adultes sur les dix-neuf que compte le village, elle travaille. Depuis sept mois, elle se rend tous les matins à l'hôtel voisin où elle est employée comme femme de ménage. Satisfaite de pouvoir gagner sa vie et ravie de "la bonne ambiance" qui règne dans l'entreprise, cette mère de six enfants lance, rieuse : "C'est bien, ce travail, ça me permet de faire un peu de sport, ça me fait maigrir" $1{ }^{27}$.

\section{- Le marquage de l'ethnicité par le genre}

Dans la presse, les adultes roms sont représentés comme ne partageant pas l'horizon d'attentes normatives de la société française. Sales, pauvres, contraints de mendier, ils sont également décrits comme ayant des comportements déviants. Les hommes roms sont notamment dépeints comme ayant une masculinité "viriliste", comme étant violents et agressifs à l'égard des autres membres de la collectivité et plus particulièrement des enfants. Libération $a$, par exemple, mis en avant "l'histoire de Daniel, 18 ans ", présentée comme "édifiante sur les relations compliquées qu'entretiennent de nombreux jeunes Roms et leur famille. Avant d'arriver au foyer, Daniel travaillait chez un poissonnier à Aubervilliers. Son père y surgissait régulièrement pour lui piquer sa paye et lui flanquer au passage une bonne rouste $11^{28}$.

La description négative des hommes a été particulièrement portée par Le Figaro à l'été 2010, lorsque ce journal a donné la parole à des personnalités politiques membres de la droite parlementaire qui ont décrit, de manière privilégiée, ces migrants comme se rendant coupables d'abus et de trafics, à l'image de Pierre Lellouche, secrétaire d'État chargé des Affaires européennes, pour qui II ces populations, qui n'ont souvent jamais été scolarisées, sont dans la plupart des cas victimes de véritables réseaux et trafics d'êtres humains, qui prospèrent en contraignant des personnes âgées et des enfants à mendier dans nos rues, en livrant des jeunes filles à la prostitution ou en forçant des mineurs à des activités délictueuses $1{ }^{29}$. Cette représentation des hommes roms fait écho, d'après nos observations et enquêtes antérieures, à la construction médiatique d'autres groupes minoritaires définis dans la presse par des identités de genre déviantes : le "jeune garçon des

27. ANDRÉ, Pauline, "À Aubervilliers, la caravane trépasse", Libération du 29-7-2010, http://www.liberation.fr/societe/2010/07/29/a-aubervilliers-la-caravane-trepasse_669130

28. TASSEL, Fabrice, "17 ans, Rom et en quête d'insertion", Libération du 28-9-2012, http://www.liberation.fr/societe/2012/09/27/17-ans-rom-et-en-quete-d-insertion_849363

29. LELLOUCHE, Pierre, "Roms : la liberté de circuler, le devoir d'intégrer", Le Figaro du 24-8-2010. 
banlieves", le "père immigré" et l'"islamiste" constituent en effet trois stéréotypes incarnant les "non-Blancs" dans la presse, catégorisés en l'occurrence par une masculinité exacerbée ${ }^{30}$.

Dans leurs comportements, les enfants et les adolescents, quel que soit leur genre, ont été mis en scène comme étant différents des membres masculins plus âgés du groupe. En effet, ils ont été construits comme victimes de violences et de pressions du fait de leur âge et de l'ascendant des hommes. Une double étiquette de coupables et de victimes est ainsi accolée à ces populations, distribuée différemment selon leur âge et leur genre.

Contrairement aux hommes, les femmes ne sont pas mises en scène en tant que coupables, mais comme étant d'abord soumises à l'autorité de leur mari et à celle de la collectivité. Elles ont été représentées comme ayant été précocement mariées, parfois à la suite de négociations et d'arrangements familiaux, à l'image de cet article publié dans Le Monde: "Une des grandes satisfactions de Mihaela Popoiu [directrice de l'école de Tarlungeni ${ }^{31}$ ] est la diminution du nombre de jeunes filles mariées. La première, il y a quatre ans, avait 14 ans; la seconde, l'an passé, en avait 15. "Il ne s'agissait pas de mariages arrangés, les deux filles étaient amoureuses, soupire la directrice. On a essayé de faire une médiation, mais c'était trop tard. On a juste obtenu qu'elles poursuivent leurs études après le mariage" $11^{32}$.

Les femmes sont mises en scène comme vivant dans un schéma conjugal traditionnel : assujetties à leur époux, elles élèvent de nombreux enfants et sont contraintes à la reproduction, comme l'a dépeint ce journaliste du Monde : "Une des filles de Daniela [une Roumaine de 46 ans habitant à Tarlungeni, dans une maisonnette à la lisière du quartier rom], mariée à un Rom, en est à son cinquième enfant. "Elle veut arrêter, mais lui refuse, car c'est dans la tradition rom d'avoir le plus d'enfants possible" soupire-t-elle $1{ }^{33}$. Les femmes roms sont plus particulièrement présentées comme ne partageant pas les normes de genre de la société française et ses idéaux d'“émancipation". Souvent désé-

30. Cf. DALIBERT, Marion, "Quand le genre représente la race. Les processus d'ethnoracialisation dans la couverture médiatique de Ni putes ni soumises", in : DAMIAN-GAILLARD, Béatrice ; MONTANOLA, Sandy; OLIVESI, Aurélie (sous la direction de), L'assignation de genre dans les médias : attentes, perturbation, défigements, Rennes: Presses universitaires de Rennes, 2014, pp. 43-54

31. Village roumain de 2700 habitants.

32. SMOLAR, Piotr, “Un mur en 'Tziganie'”, Le Monde du 16-8-2010, http://www.lemonde.fr/ europe/article/ 2010/08/14/ un-mur-en-tziganie_1399020_3214.html

33. Ibidem. 
rotisées, leur figure peut être rapprochée de celle de la "mère immigrée" maghrébine que nous avons observée dans la médiatisation de $\mathrm{Ni}$ putes ni soumises ${ }^{34}$. Une journaliste du Monde a d'ailleurs mis en lien ces deux identités minoritaires comme suit : "I En discutant avec ses copines d'école d'ascendance algérienne, Dana a découvert que certaines "traditions", présumées communautaires, comme l'obligation faite aux filles d'arriver vierges au mariage, n'étaient pas propres aux Roms. Elle se garde de conclure. À l'ordinaire, elle s'habille comme les filles de son âge : un jean — un "slim", corrige-t-elle — un tee-shirt et un gilet noirs. Quand elle va voir les Roms, seule ou avec sa mère, laquelle entretient les contacts avec la communauté de Montreuil, elle met une jupe, "par respect pour les anciens", dit-elle, sérieuse 1135 .

Ainsi, les hommes et les femmes d'âge adulte mis en scène dans le corpus étudié ont été catégorisés par une identité de genre qu'on peut qualifier de déviante. Ce rôle joué par la représentation du genre dans les processus de catégorisation n'est pas nouveau ${ }^{36}$. Ces catégorisations genrées construisent les frontières entre groupes sociaux dans les médias : le genre devient un marqueur d'identité qui détermine et renforce l'altérité en même temps qu'il essentialise et naturalise les comportements.

\section{Le "nous" face aux Roms : anti-tsiganisme et nouveaux racismes européens?}

Les rapports - et notamment les mauvais rapports - entre les Roms et d'autres populations ont été très abondamment décrits dans la presse. Or, alors même que la France a été fortement critiquée par l'Union européenne pour les mesures prises à l'endroit de ces migrants, les membres de la "communauté nationale" n'ont jamais été mis en cause dans les médias comme pouvant être hostiles ou discriminants à leur égard. C'est bien souvent à l'étranger que les figures de l'anti-tsiganisme et les individus qui les incarnent sont à

34. Cf. DALIBERT, Marion, "Quand le genre représente la race. Les processus d'ethnoracialisation dans la couverture médiatique de Ni putes ni soumises", art. cité.

35. SIMON, Catherine, "Roms : la vie devant soi", Le Monde du 13-7-2010, http://www.lemonde.fr/ societe/article/ 2010/07/12/roms-la-vie-devant-soi_1386876_3224.html

36. Il fut même central à certaines époques et dans certains contextes politiques. Voir, par exemple, les travaux sur la colonialité de STOLER, Ann-Laura, "Genre et moralité dans la construction impériale de la race", Actuel Marx, n 38, novembre 2005, pp. 75-101; PARIS, Myriam ; DORLIN, Elsa, "Genre, esclavage et racisme : la fabrication de la virilité", Contretemps, $n^{\circ} 16$, janvier 2006, pp. 96-105. 
rechercher : I'ltalie, la Hongrie et la Roumanie ont été ici décrites comme les "mauvais élèves" européens. Les Italiens, notamment, ont été les ressortissants d'un pays membre de l'Union européenne qui ont été les plus régulièrement présentés comme racistes et discriminants, plus particulièrement au cours de l'été 2008.

Libération a ainsi rendu compte des peurs ressenties par les Roms face aux violences des Napolitains : " Ce jour-là, le chef du camp, Dragan Alexic, 44 ans, a gardé ses quatre enfants à la maison, de crainte des attaques xénophobes. "Ils ne vont pas à l'école en attendant que ça se calme", explique-t-il. Nous-mêmes, adultes, évitons pour le moment d'aller à Naples. Tous les yeux sont sur nous. Alors, on fait profil bas $11^{37}$. Les habitants de Naples ont été mis en cause à plusieurs reprises, comme on peut le constater dans un autre article du journal: II II [un Rom] dénonce l'agressivité renforcée de la police et l'hostilité croissante d'une partie de la population. En mai dernier, à Naples, un campement a été incendié et nombre de nomades ont préféré quitter la ville. Mardi, un autre regroupement de Roms a été attaqué, à Rome cette fois, dans le quartier de La Magliana, à quelques kilomètres de Corviale $11^{38}$.

En Hongrie, les sentiments xénophobes portés par les mouvements fascistes - mais décrits comme recevant "l'appui de la troisième force politique au Parlement, le Jobbik, [ainsi que] de certains conservateurs $11^{39}$ — ont également été soulignés. Enfin, la Roumanie a été présentée comme le pays responsable des conditions de vie misérables des Roms, plus particulièrement par Le Figaro. Ce journal a fortement relayé ce discours énoncé par des politiques ou d'autres acteurs sociaux à l'été 2010 , en réponse aux multiples accusations portées contre les membres du gouvernement de François Fillon. JoëlBenoît d'Onorio, juriste et professeur des universités, a par exemple expliqué dans ce quotidien : " Chez nous, la sécurité (c'est-à-dire le bienêtre) et les ressources font défaut à ces populations car l'État français n'a pas les moyens de faire plus qu'il ne fait déjà et qu'on exige néanmoins qu'il fasse en lieu et place de la Roumanie qui se permet encore de s'en indigner ! C'est pourtant bien là-bas et nullement ici que se trouvent la cause de leur misère et la solution de leur avenir $11^{40}$.

37. MASCIARELLI, Alexis, "À Naples, les Roms craignent de nouvelles attaques", Libération du 46-2008, http://www.liberation.fr/monde/2008/06/04/a-naples-les-roms-craignent-de-nouvellesattaques_73287

38. JOZSEF, Éric, "Silvio Berlusconi compte ses Roms", Libération du 24-7-2008, http://www.liberation.fr/ jour/2008/07/24/silvio-berlusconi-compte-ses-roms_76903

39. STOLZ, Joëlle, "Nouvelle poussée de fièvre extrémiste en Hongrie", Le Monde du 3-9-2012.

40. ONORIO, Joël-Benoît d', "Le Pape, les Roms et la laïcité", Le Figaro du 28-8-2010. 
Contrairement aux Italiens, aux Hongrois ou aux Roumains, les Français ne sont guère mis en cause, de manière collective, comme étant coupables d'attitudes racistes ou xénophobes. À l'exception des membres du gouvernement Fillon et du président Nicolas Sarkozy dont les actions furent fortement critiquées, c'est la responsabilité d'autres populations minoritaires ou d'individus venant d'un milieu social modeste qui a été mise en avant dans la presse. Un certain nombre d'articles rendant compte des discriminations à l'égard des Tsiganes en France les fondent sur les expériences rapportées des banlieues pauvres de Marseille. Un journaliste du Monde s'arrête ainsi sur l'incident qui oppose II une trentaine d'habitants et riverains de la cité des Crénaux, dans le $15^{\mathrm{e}}$ arrondissement de Marseille " et " des familles roms installées à proximité " : " Une trentaine de Roms étaient installés sur ce campement depuis quelques jours. Révélée par La Provence, cette expédition punitive était préméditée. Ces habitants avaient informé les autorités, dont une élue des quartiers Nord, de leur passage à l'acte si les Roms ne quittaient pas les environs de la cité ${ }^{4}{ }^{41}$.

Ces situations ont toutefois été construites comme étant plutôt des exceptions face à l'attitude des nationaux, généralement dépeinte comme juste et égalitaire, à l'image de cet extrait d'article paru dans Le Monde: "Experts dans l'art de la débrouillardise, les Bita se nourrissent des produits périmés trouvés dans les poubelles des supermarchés. "Puis il y avait les Restos du cœur, dit-elle. Les Français ont un grand cœur. Ils nous ont traités mille fois mieux qu'en Roumanie". Le centre d'accueil municipal de Grenoble les aide à passer l'hiver à l'hôtel Paris-Nice $1{ }^{42}$. Les qualifications positives auxquelles les "Français" ont été associés ont d'ailleurs souvent été mises en scène comme étant portées par les Roms eux-mêmes, avec donc un effet d'authentification et de légitimation de ces discours définitoires lié à l'usage de la citation ${ }^{43}$.

Ces dimensions internationales et européennes formulées à partir de l'étude de la presse acquièrent une résonance particulière lorsqu'elles sont mises en perspective, comme nous proposons de le rappeler rapidement ici, avec des analyses plus politiques et sociologiques

41. AFP, "Des habitants d'une cité de Marseille chassent des Roms et mettent le feu à leur campement", Le Monde du 29-9-2012.

42. RICARD, Philippe; VINCENT, Élise, "Les expulsions de Roms pèsent sur la rentrée européenne de Nicolas Sarkozy", Le Monde du 16-9-2010,

43. Cf. LAROCHE-BOUVY, Danielle, "Émergence de l'interaction verbale dans la presse écrite : fonction de la citation", in : CHARAUDEAU, Patrick (sous la direction de), La presse : produit, production, réception, Paris : Éd. Didier Érudition, 1988, pp. 113-130. 
des processus de construction communautaire et de leur incidence sur les migrations et les destinées de ces populations. En effet, comme le souligne Étienne Balibar, dans une Europe en élargissement, la construction publique du "problème rom" ou de la "question rom" est souvent révélatrice de tensions et de contradictions. Celles-ci mettent à l'épreuve le projet et les principes mêmes de l'Union : " $\dot{A}$ tout le moins, l'unification de l'Europe a rendu plus visible le processus de racisation du problème tsigane du fait même qu'il contredit brutalement sa prétention officielle à pousser comme telle à l'extinction des préjugés ethniques $1{ }^{44}$. Si les nations européennes ne se haïssent plus, consciemment du moins, beaucoup de préjugés et de ressentiments ne demanderaient qu'à ressurgir. Les populations dites "tsiganes", par leurs trajectoires de mobilité et de déterritorialisation, se trouvent être une cible privilégiée pour le déplacement et la cristallisation de ces sentiments nationalistes intra-européens. Perçues comme une II nation en trop ", stigmatisées parce qu'll à cheval " sur les frontières, formant "I le type-même de population sans État, résistant à la territorialisation et à l'homogénéisation culturelle ${ }^{45}$.

Ces nouvelles formes de préjugés, de dénonciations et de discriminations, si tant est qu'elles soient inédites, s'ajoutent et actualisent les répertoires traditionnels de l'anti-tsiganisme. Comme le rappellent en France les travaux de Jean-Pierre Liégeois, la République aura eu en la matière peu d'impact correctif ${ }^{46}$; plutôt que de combattre les stéréotypes ancestraux contre les Bohémiens, les républicains les institutionnalisent à travers des mesures applicables aux "nomades" et aux "voyageurs". Gommant explicitement la dimension ethnique, ces catégories se caractérisent par une double réduction: d'une part, au fait de ne pas posséder de bien immobilier et, d'autre part, à l'exercice de certaines activités professionnelles, ce qui vient à former des désignations " ethnico-professionnelles ", mais aussi " des catégories discriminatoires légales ${ }^{47}$. Les rapprochements opérés aujourd'hui entre les populations immigrées en provenance des pays

\footnotetext{
44. BALIBAR, Étienne, "Racisme et politique communautaire : les Roms", in : CANUT, Cécile (dossier coordonné par), "L'exemple des Roms. Les Roms, pour l'exemple", Lignes, n³4, février 2011, pp. 135-144 (voir p. 140).

45. Ibidem, p. 141.

46. Cf. LIÉGEOIS, Jean-Pierre, Roms et Tsiganes, Paris : Éd. La Découverte, 2009, 125 p.

47. BLUM LE COAT, Jean-Yves; CATARINO, Catherine; QUIMINAL, Catherine, "Les gens du voyage : errance et prégnance des catégories", in : GOTMAN, Anne (sous la direction de), Villes et hospitalités: les municipalités et leurs "étrangers", Paris : Éditions de la Maison des sciences de l'homme, 2004, pp. 157-176.
} 
de l'Est et la catégorie a priori désethnicisée des "gens du voyage"48 démontrent bien une logique racisante et essentialiste à l'œuvre dans leur perception et leur traitement.

S'agit-il toutefois de nouvelles formes de racisme qui prolongent par rapport à la construction de l'ensemble politique supranational ce que les racismes "classiques" (antisémitisme, racisme colonial) auraient représenté par rapport à l'État-nation ou est-ce la permanence d'une interprétation raciale qui fait toujours autorité "bien plus qu'on ne veuille le reconnaître ${ }^{49}$, l'unité "raciale" des Roms, surtout lorsqu'on voyage à l'Est, se mesurant d'abord, comme le rappelle Alain Pierrot, à la couleur de leur peau?

Comme le montre notre étude de la représentation médiatique, la problématique des rapports de pouvoir à l'égard des Roms est restée à l'ombre de la sphère publique : dans le discours médiatique, les membres de la "communauté nationale" ont été construits comme étant intrinsèquement égalitaires, alors que c'est une vision essentialisée et racialisée de l'Autre qui a été proposée, confinant ces populations dans une forme d'altérité radicale et naturalisée.

Que ce soit à travers l'assignation territoriale ou les représentations genrées, ce marquage opère dans la sphère publique médiatique en écho aux discours et débats plus politiques qui mettent en avant leur "indésirabilité" ou leur "incapacité" de s'intégrer et légitiment les politiques d'expulsion. Dans les médias, ce processus d'altérisation conduit paradoxalement à la construction d'un "groupe sans visage", voire à une forme de déshumanisation, attendu qu'il signifie aussi un déni de reconnaissance, générateur à son tour d'indifférence et d'insensibilité accrues ${ }^{50}$. Les représentations de la marge autorisent les poli-

48. Cf. COSSÉE, Claire, "Les-Roms-migrants-et-gens-du-voyage”, Lignes, n 35, juin 2011, pp. 167-179.

49. PIERROT, Alain, "Mythe nomade et logique migratoire", in : CANUT, Cécile (dossier coordonné par), "L'exemple des Roms. Les Roms, pour l'exemple", Lignes, n³4, février 2011, pp. 52-78 (voir p. 73).

50. Cf. BUTLER, Judith, Humain, inhumain: Ie travail critique des normes. Entretiens, Paris: Éd. Amsterdam, 2005, 154 p. 
tiques de l'exception entreprises à son égard, avec leur lot de stigmatisations, de préjugés, de dénonciations et de discriminations qu'il s'est agi ici de rappeler.

L'analyse montre également comment cette représentation des minoritaires participe en creux de la construction d'un discours et d'un "nous" national, interpellé en l'occurrence tant par la vision politique d'une Europe "sans frontières" que par les registres plus locaux de la ségrégation et des racismes ordinaires. 